\title{
THE DESIGN STUDIO AS A NETWORK - APPLICATION OF CONNECTIVISM IN THE PLANNING OF AN ONLINE LEARNING SPACE
}

\author{
Miroslava Nadkova PETROVA \\ Universidad de Monterrey
}

\begin{abstract}
The design studio is the core element in the design curriculum where students gain key knowledge and skills. When the traditional classroom course had to be transferred to an online modality enforced by covid-19 pandemics, re-conceptualization of the structure and contents was required in order to ensure the quality of the teaching and students' satisfaction. Based on the premise that the contents should not be simply adapted to an online version, but an entirely new learning experience should be created, the redesign of the class was inspired by the principles of connectivism (Siemens, 2005). Connectivism as an alternative learning theory recognizes the societal shifts and the inevitable impact of technology on the learning processes. This new framework for understanding learning, states that knowledge is derived externally of the individual through a process of connecting nodes and patterns recognition. These abilities are considered especially important to prepare design students for the challenges of the profession within the context of the fourth industrial revolution.

The paper explores the potential of connectivism as a holistic approach to the planning of the contents of an online design studio. It describes the process of designing the course structure and discusses the challenges encountered in the creation of a connectivist learning environment. The advantages of the networked design studio were verified in a survey on the perceptions of the students in regard to their satisfaction and the effectiveness of their knowledge acquisition.
\end{abstract}

\section{Keywords: Connectivism, design studio, online education}

\section{INTRODUCTION}

Design education has undergone significant development in the past decades to respond to the technological and social changes and to adequately prepare students for their future professional practice. Digital technology has been extensively embraced by the educational system to facilitate the learning process and to improve the quality and effectiveness of the teaching. Enabled by Web 2.0 online learning brought a radical change in the educational context with its accessibility, independence of time and space and the ability to promote varied interactions with the content, the instructor and the other learners. However, the giant leap to online learning was not a natural result of its advantages but was externally imposed by the covid-19 global pandemic when it proved to be the only possible way for the educational process to continue. University educators were faced with the fact that in the changed setting learning occurs in a different way. They had to rapidly adapt and discover the new opportunities for teaching and learning afforded by the online environment. I also had to re-evaluate the content of the studio classes I teach and to plan how to present and transform the information in a relevant way. I was aware that to ensure the quality of my teaching and to respond to the needs and expectations of my students, the contents cannot be simply transferred to the online version of the class. Instead, I had to fundamentally rethink my teaching approach and to devise entirely new experience for the students. Guided by the principle summarized by Laurillard that "[k]nowledge technologies shape what is learned by changing how it is learned,"[1] I aimed to provide a systematic organization of the content and to plan the class interaction by combining the best pedagogical practices of the traditional face-to-face class with the emerging possibilities provided by the virtual environment. 


\section{TOWARDS A VIRTUAL DESIGN STUDIO}

The design studio is the core element in the design curriculum where students gain key knowledge and explore their creative skills [2]. By implementing a project-based approach, the studio is where students "express and explore ideas, generate and evaluate alternatives, and ultimately make decisions and take action" [3]. Students learn how to communicate their concepts through representation, how to master the visual design language, or generally said they learn how to design [4]. The pedagogical approach is focused on the following three educational aspects - new skills, new language and the development of student's thinking [5]. However, central characteristic of the studio is not pedagogy but the interactions that take place and which form the exemplary atmosphere or the studio culture. Wang describes it as "a vital complex of material representation, social collaboration, creativity, emotionality and a tolerance for uncertainty - if not outright confusion - balanced with a faith that meaningful designs eventually will emerge." [6]. Through the critical dialogue that is established and the provided feedback, students tests the validity of their ideas, and constantly revise and improve them. In this way knowledge acquisition occurs - in the experiential creative process defined by Schön as "reflection-in-action" [7]. The traditional notion is that the social and cultural domain of the studio has a counterpart in the physical environment where students spend long hours, create their own community of practice, and identify with it as individuals and as a group [8]. However, with the pervasion of information and communication technologies both in the design practice and the educational process, the design studio was inevitably transferred to the digital realm. Driven by the growing complexity of the design problems and the higher demand for collaboration between all participants involved in the design process, in the early 1990s the virtual design studio emerged [9]. The new studio typology offers a computer-mediated collaboration, often between geographically distributed and multicultural teams which is space and time independent [10]. Though considerably different from the physical space, the virtual studio has the same function as a shared learning and practice space where students interact, develop and present their projects. Furthermore, an important fact is that a sense of place can be fostered [11] and hence the identity and the community typical for the face-to-face studios can be successfully retained. A major benefit of the experience in a virtual design studio is that students are exposed to a simulation of the real working environment where expertise in digital media and collaboration in multidisciplinary teams are prerequisites. In the virtual studio students master the new digital tools and develop competences which help in bridging the gap between academia and the professional practice.

\section{THEORETICAL FRAMEWORKS FOR LEARNING IN THE DIGITAL AGE}

To understand the effects of the technological context on education and to support the planning of the online design studio a relevant theory is needed. Determinant factor to be considered is the changing nature of the design process. The commonly accepted notion of its structure consists of two situations an identified problem that needs to be resolved and a solution which fulfils a certain goal. The transformation from one state to the other, or the causal link between the two situations, is the act of design [12]. From the perspective of systems and complexity theories, Findeli suggests that instead of a problem and a solution, two end states of the system should be considered - its present state and its desired future state which is never a specific solution but a transitory state in a dynamic process. In this new structure the designer and the user are also considered parts of the system that undergo changes during the transformation process. The awareness of the systematic nature of design requires attention to be paid to the invisible relations that exist between the actors within the system and not on the artifact as an outcome of the design project. This new understanding might be the radical change needed in design education and the design studio in particular which are in a state of crisis [6].

Our students, the future designers need to be trained how to deal with the increasing complexity of both the design problems they have to resolve and the design process which often requires a multidisciplinary approach to the design project. To support the acquisitions of skills and prepare them for the challenges of the profession within the context of the fourth industrial revolution, learning should not be focused on the accumulation of knowledge but on the ability to seek for the most up-to-date information, to filter it and to apply it when making decisions. Again, the importance of the relations within the highly abundant and rapidly changing information network and the ability to explore them is prioritized. Hence, the application of systems and complexity theory in learning can provide the demanded framework and foster the required change in education.

In his seminal work "Connectivism: A Learning Theory for the Digital Age" Siemens questioned the viability of behaviourism, cognitivism and constructivism as theories adequately addressing the learning 
processes in the digital age [13]. He advanced connectivism as an alternative learning theory recognizing the societal shifts and the inevitable impact of technology on learning processes. He reconsidered the relationships between knowledge and the learners in the current social environment and proposed a novel understanding that knowledge is derived externally of the individual through a process of connecting nodes and patterns recognition. According to connectivism, knowledge is a network phenomenon, composed of networked entities and their connections [14]. A major tenet is that knowledge is considered as a function of elements distributed across a system; it is decentralized and may exist outside of a person, which implies that "know where" becomes more important than to "know what" and to "know how." Learning is considered as a continual network-forming process in which knowledge is created through the construction of new connections between fields, ideas and concepts. It evolves from knowledge consumption to a knowledge creation process [15].

Though the status of connectivism of a theory has been criticized [16], [17], its conceptualization of learning accurately reflects the context defined by the online learning environment and the changed student demographics, so I consider its application as a pedagogical approach in the design studio pertinent.

\section{CONNECTIVIST LEARNING IN THE ONLINE DESIGN STUDIO}

The studio takes a central place in the four-year undergraduate programme of Interior Design at the University of Monterrey, Mexico. From the very beginning of the course of study, students are exposed to studio work which each subsequent semester features a changing focus of the subject and increasing complexity. Successful completion of the previous studio is required for the students to enrol in the next studio and to continue their studies. The class in consideration which had to be transformed from a traditional studio into a fully online mode in the autumn semester in 2020 is "Studio Integral." This is the last and most advanced studio which allows students to apply progressive theoretical approaches in the development of a comprehensive large-scale project.

The major questions considered in the planning process of the online studio were:

- How to plan for effective communication and collaboration in the online learning environment?

- How to engage students and how to encourage a more autonomous and self-directed learning?

- How to ensure meaningful knowledge acquisition in the online learning environment?

- How to support new types of interaction so that the authentic culture and practice of the design studio is preserved in the online learning environment?

The first aspect taken into account was the profile of the students. The impact of technology on millennial students' behaviour, beliefs, attitudes and educational requirements has long been recognized and researched with the intention to implement learning activities which are meaningful and conform to their learning needs [18]. However, in 2020 a new digital native generation is in the classroom and though sharing some characteristics with their predecessors, they certainly have their differences. To understand the characteristics and the learning preferences of the cohort it is imperative to support the students through their learning journey and to maximize their engagement in the educational process. Generation Z students are described as observers with a preference for visual and video-based content. They identify themselves as intrapersonal learners who are used to learning in their own setting before sharing their knowledge with others. They focus on acquiring the skills needed for their future career and expect to immediately apply them in real life. Moreover, they feel highly motivated when they are engaged with their passions and when they are involved in social change initiatives [19].

A main goal of the design of the teaching strategy was not to focus on the content but to align the learning outcomes with the proposed activities so that each student is enabled to master competencies while comprehending the value they add for his professional development.

The expected learning outcomes were formulated as follows:

- $\quad$ Ability to implement systematic design approach to solving problems

- Skills to develop and structure a design narrative as a prerequisite for a successful design solution

- Ability to translate conceptual ideas into tangible forms and spaces

- Analytical and critical thinking skills for generalization, evaluation and selection of structures, constructions and materials

- Mindset to apply integrative design approach for sustainable solutions

- Presentation skills and ability to defend concepts and ideas with well-grounded arguments

- Interdisciplinary and heuristic thinking 
Contrary to the departmental requirement that the course content should be well-defined and preliminary structured, I proposed a flexible programme which could easily adapt to changes and self-organize according to the needs of the students.

Following connectivist principles I tried to offer a variety of perspectives and opportunities for students to connect and to establish dialogue. I relied on the interactions which would be naturally formed and the spontaneous emergence of learning through these interactions. The didactic content was not hierarchically structured but instead was split into small segments which could be easily re-arranged and personalized according to the prior knowledge and individual interest of the students. The theoretical lectures and videos supporting the concepts which were to be explored in the design process were uploaded on the learning management system for students to review at their own pace. At the same time, students were encouraged to research these concepts further, to collect reference materials and through reflexion and self-critique to move from observation to interpretation.

A major premise in this teaching strategy is that the students are responsible for their own learning. However, in general, they are accustomed to receiving precise instructions and prefer to be guided in the development of the assignments. To prevent them from getting lost in the massively abundant information and to create a feeling of security, the synchronous sessions in the beginning of the semester were devoted to creating an atmosphere of trust and confidence. The provided nodes of theoretical content served as guidelines for the initial building of a personal knowledge base while the online discussion forums resolved doubts and supported students in the process of self-directed autonomous learning (Figure 1). The formation of this knowledge base is critical for the acquisition of skills to recognize which information is valuable and authentic.

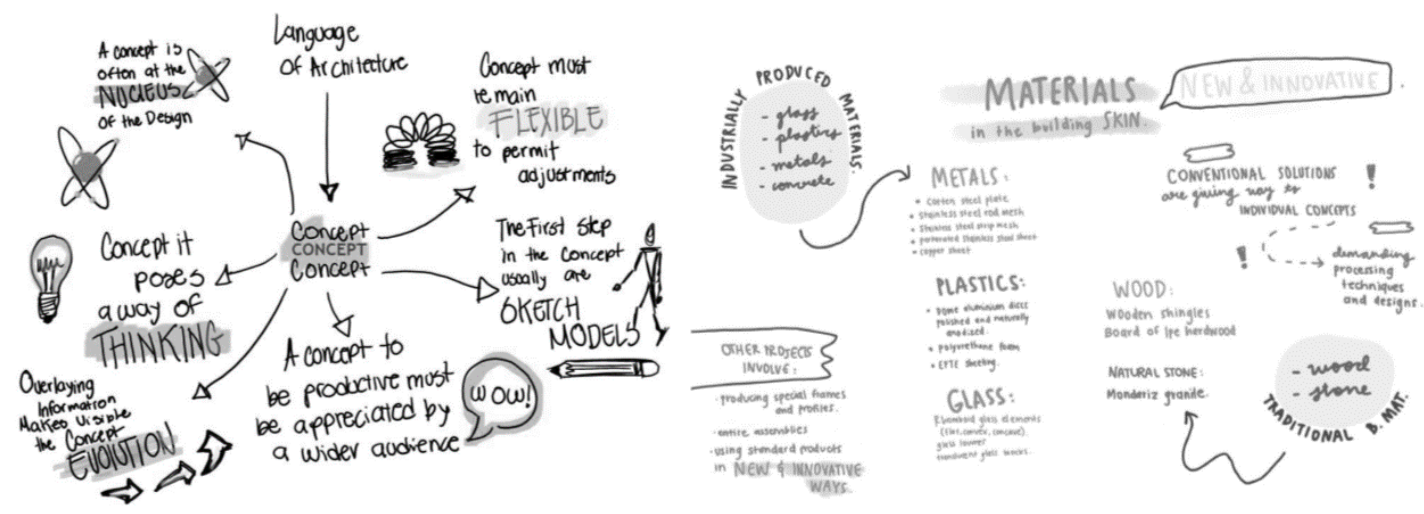

Figure 1. Mind-maps representing student's understanding of the theoretical concepts

When understanding of the basic concepts has been developed, students could move on to the next level where through interactions with a new content they could form new connections. The formation of new and unexpected connections between existing ideas and the most up-to-date concepts results in new forms of knowledge and this is the essence of the creative process. Not only analytical and critical thinking but also interaction and discussion are crucial for students to be able to "see" these new connections (Figure 2).

The feedback provided by peers and the instructor aided in distinguishing the valuable inputs. Another important aspect is that students are expected to contribute to the knowledge network by sharing insights and disseminating their own knowledge. Engagement and active participation of each student is expected. In this knowledge co-creation process the main role of the teacher is to curate and sustain the learning environment, to monitor and control its effective functioning, to motivate the self-directed learning and to create opportunities for knowledge sharing. One such opportunity was provided with the implementation of a collaborative online international learning (COIL). This virtual mobility experience made possible the interaction with students from another country (Ecuador in this case), gave a new contextual meaning to the explored ideas, encouraged cross-national dialogue and additionally expanded the created networks in a culturally diversified environment. 

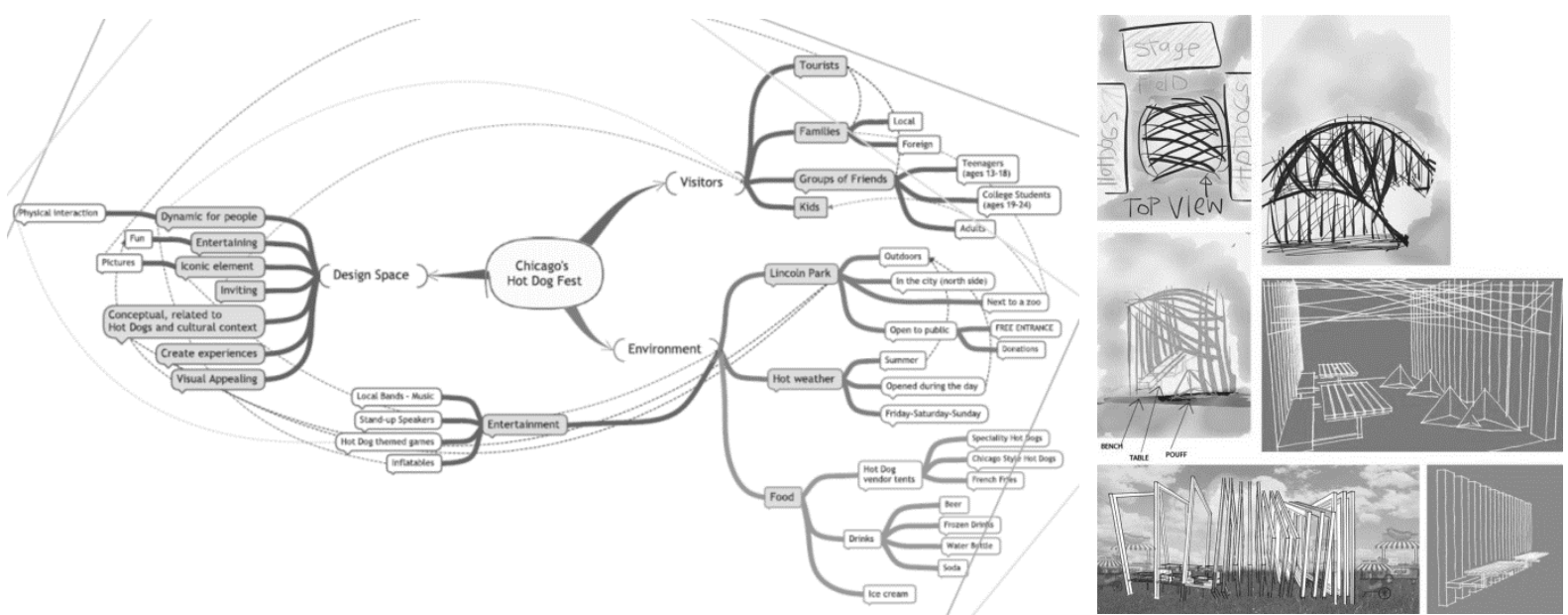

Figure 2. Mind-map exploring the existing relationships between the major aspects considered in a pavilion design and the resulting concept sketches

\section{LESSONS LEARNED}

The perceptions of the students and their own estimation about the achieved outcomes of the class were examined with several surveys which were carried out before, during and at the end of the semester. In this way I could verify the effectiveness of the teaching strategy but also gain valuable recommendations and critical feedback on what to change and how to improve the online learning environment. One of the most satisfying results was that all 15 students found the class to be very well planned and would not change anything in the future. Also, all students replied positively to the question "Did you find the learning in the studio meaningful?" Another survey question addressed the perception of the level at which the class prepares students for their professional practice. 53\% indicated a "very high" level, and the other $47 \%$ evaluated the level as "high." Likewise, all students considered that their personal achievements in the class as "very high" which was also confirmed with my direct observations.

However, in the answers to the question whether the online studio can replace the face-to-face classes prevail the opinion that traditional classes are indispensable as $67 \%$ preferred them. Among the reasons were pointed out that communication is better in person than talking to a camera, because working in front of the computer for long hours has been very tiring, and distractions occur more often at home. Still, students found a lot of advantages of the online studio. A respondent commented, "I liked the class very much, I think that the teacher understands that the online class is harder for us, but she makes it very interesting and useful, also not that difficult and always supports us." Another student shared, "Yes, I think the proposed type of learning made us more responsible. We, as individuals, are responsible of our own learning. Learning goes way further than connecting to the class, we have to be present and have the best attitude in order to be able to learn". Another opinion which summarizes the advantages of the teaching strategy is, "This class has helped me improve my level of organization and my design process."

I consider an indicator of the success of the proposed teaching strategy to be the changed attitude of the students towards the responsibility for their own learning. While at the beginning of the semester $80 \%$ responded that they prefer to receive precise instructions how to develop the assignments and only $30 \%$ were willing to search additional references and readings not assigned by the teacher, at the end of the semester 14 of the students defined themselves as self-directed learners and only one was uncertain. The feeling of belonging to the community was also confirmed by $93 \%$ of the students.

One of the findings which require some changes to be implemented in the future is that $73 \%$ of the students think that the overload of the online class is higher than the traditional face-to-face class and all of them felt very tired at the end of the semester.

I was surprised by a comment from one of the students who shared, "Thank you for caring about our experience with online classes. This is the first time a professor takes the time to ask important questions about this type of learning." I find this a big shortcoming because educators should always be aware of the effectiveness of their classes as there is a direct relation between the selected method of teaching and the learning outcomes. And this is especially important to be identified in the online environment which most likely is completely new for the majority of the teachers. 


\section{CONCLUSIONS}

The creative transformation of the traditional studio class into a highly interactive and meaningful online environment was considered a major prerequisite for ensuring students' satisfaction and valuable learning experience. Through the implementation of the connectivist learning strategy I was not only able to adapt to the new circumstances but to offer a space with added value where students were able to fully unfold their creative potential. Though still being highly controversial and criticized, connectivism answered my need to translate the content of the face-to-face studio class and to convert it into an advanced knowledge network. My major objective was to encourage students to be critical, to search for problems in the world around us, to learn from other fields and cultures, to create narratives which merge poetry and advanced technology and to aid them in the process of becoming visioners of a better future. The positive results of the survey unambiguously showed that the initial frustration created by the lack of rigid structure and higher uncertainty of the studio organization was overcome and to a certain extent students learned how to deal with complexity.

\section{REFERENCES}

[1] Laurillard D. Teaching as design science. Building Pedagogical Patterns for Learning and Technology, 2012 (Routledge).

[2] Salama A. New Trends in Architectural Education: Designing the Design Studio, 1995 (ARTI$\mathrm{ARCH})$.

[3] Gross M. and Do E. The Design Studio Approach: Learning Design in Architecture Education, in J. Kolodner \& M. Guzdial [Eds] Design Education Workshop, EduTech/NSF, College of Computing, Georgia Institute of Technology, September 8-9, 1997.

[4] Corona-Martínez A. The Architectural Project, 2003 (Texas A\&M University Press) Ledewitz, S. Models of Design in Studio Teaching. Journal of Architectural Education, 38(2), pp. 2-8, 1985.

[5] Ledewitz S. Models of Design in Studio Teaching. In Journal of Architectural Education, Vol. 38(2), pp. 2-8, 1985.

[6] Wang T. A New Paradigm for Design Studio Education, Journal of Art and Design Education, 29.2, pp.173-183, 2010 (NSEAD/Blackwell Publishing Ltd).

[7] Schön D. The Architectural Studio as an Exemplar of Education for Reflection-in-Action. Journal of Architectural Education, 38(1), pp. 2-9, 1984.

[8] Spruce J. Examining the Role of the Studio Environment within Design Education, In Proceedings of the International Conference on Engineering and Product Design Education, New Castle upon Tyne, UK, 2007.

[9] Radojevic M. Codification of Site Related Knowledge in Virtual Design Studios. In Design Studio Pedagogy: Horizons for the Future [Eds] A. Salama \& N. Wilkinson, 2007 (ARTI-ARCH).

[10] Maher M., Simoff S., and Cicognani A. Understanding Virtual Design Studios, 2012 (Springer).

[11] Maher M. and Simoff S. Variations on the virtual design studio. In Proceedings of fourth international workshop on CSCW in design, pp. 159-165, 1999.

[12] Findeli A. Rethinking Design Education for the 21st Century: Theoretical, Methodological, and Ethical Discussion. In Design Issues Vol. 17 (1), pp.5-17, 2001.

[13] Siemens G. Connectivism: A learning theory for a digital age. International Journal of Instructional Technology and Distance Learning, 2(1), pp. 3-10, 2005.

[14] Downes S. Learning networks and connective knowledge. In Collective intelligence and ELearning 2.0: Implications of web-based communities and networking, pp. 1-26, 2010.

[15] Siemens G. Knowing Knowledge, 2006 (Lulu.com).

[16] Verhagen P. Connectivism: A new learning theory? 2006.

[17] Kop R. and Hill A. Connectivism: Learning theory of the future or vestige of the past? In International Review of Research in Open and Distance Learning, Vol.9 (3), 2008.

[18] Petrova M. Educating Designers from Generation Y - Challenges and Alternatives. In Proceedings of the International Conference on Engineering and Product Design Education, Twente, The Netherlands, 2014.

[19] Seemiller C. and Grace M. Generation Z: A Century in the Making, 2018 (Routledge). 